\title{
Multi-Level Representation Learning for Chinese Medical Entity Recognition: Model Development and Validation
}

Zhichang Zhang, PhD; Lin Zhu, MS; Peilin Yu, MS

College of Computer Science and Engineering, University of Northwest Normal, Lanzhou, China

Corresponding Author:

Zhichang Zhang, PhD

College of Computer Science and Engineering

University of Northwest Normal

967 Anning East Road

Lanzhou,

China

Phone: 8613038769329

Email: zzc@nwnu.edu.cn

\section{Abstract}

Background: Medical entity recognition is a key technology that supports the development of smart medicine. Existing methods on English medical entity recognition have undergone great development, but their progress in the Chinese language has been slow. Because of limitations due to the complexity of the Chinese language and annotated corpora, these methods are based on simple neural networks, which cannot effectively extract the deep semantic representations of electronic medical records (EMRs) and be used on the scarce medical corpora. We thus developed a new Chinese EMR (CEMR) dataset with six types of entities and proposed a multi-level representation learning model based on Bidirectional Encoder Representation from Transformers (BERT) for Chinese medical entity recognition.

Objective: This study aimed to improve the performance of the language model by having it learn multi-level representation and recognize Chinese medical entities.

Methods: In this paper, the pretraining language representation model was investigated; utilizing information not only from the final layer but from intermediate layers was found to affect the performance of the Chinese medical entity recognition task. Therefore, we proposed a multi-level representation learning model for entity recognition in Chinese EMRs. Specifically, we first used the BERT language model to extract semantic representations. Then, the multi-head attention mechanism was leveraged to automatically extract deeper semantic information from each layer. Finally, semantic representations from multi-level representation extraction were utilized as the final semantic context embedding for each token and we used softmax to predict the entity tags.

Results: The best F1 score reached by the experiment was $82.11 \%$ when using the CEMR dataset, and the F1 score when using the CCKS (China Conference on Knowledge Graph and Semantic Computing) 2018 benchmark dataset further increased to $83.18 \%$. Various comparative experiments showed that our proposed method outperforms methods from previous work and performs as a new state-of-the-art method.

Conclusions: The multi-level representation learning model is proposed as a method to perform the Chinese EMRs entity recognition task. Experiments on two clinical datasets demonstrate the usefulness of using the multi-head attention mechanism to extract multi-level representation as part of the language model.

(JMIR Med Inform 2020;8(5):e17637) doi: 10.2196/17637

\section{KEYWORDS}

medical entity recognition; multi-level representation learning; Chinese; natural language processing; electronic medical records; multi-head attention mechanism 


\section{Introduction}

\section{Background}

Electronic medical records (EMRs) comprise patients' health information. Diagnostic accuracy can be improved by making full use of the available information in EMRs. Medical entity recognition (ER) is a fundamental task of medical natural language processing (NLP) and is usually treated as a sequence labeling problem [1]. As shown in Figure 1, in which three predefined entity categories are disease, drug, and treatment, when using the BIO (beginning of the noun phrase, middle of the noun phrase, and not a noun phrase) labeling mode to tag Chinese EMRs, the candidate label set contains seven types: B-Dis (disease), I-Dis, B-Med (medicine), I-Med, B-Tre (treatment), I-Tre, and O.

Figure 1. A tagging example of Chinese electronic medical records.

\begin{tabular}{|c|c|}
\hline Sentence & Tagging Results \\
\hline $\begin{array}{l}\text { 临床初步诊断：急性支气管炎, 口服磺啶新林 } \\
\text { 胶囊。给予抗炎、解痉、祛痰对症支持治疗, } \\
\text { 亚相关检查。 } \\
\text { ( The preliminary analysis of the clinical } \\
\text { diagnosis : acute bronchitis, oral } \\
\text { sulfamethoxazole capsules . Give anti- } \\
\text { inflammatory, antispasmodic, expectorant } \\
\text { symptomatic support treatment, perfect relevant } \\
\text { examination.) }\end{array}$ & 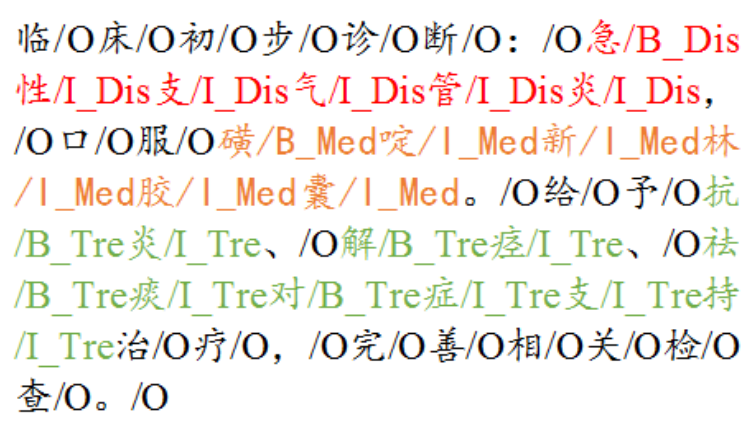 \\
\hline
\end{tabular}

Generally, the methods of ER can be divided into two categories. The first category leverages rules and dictionaries to represent linguistic features and domain knowledge to identify clinical entities [2]. The second category is based on traditional machine learning and neural networks [3-8]; this type of method greatly improves the performance of ER models but requires large-scale labeled data during model parameter training. In the medical field, creating annotation datasets is restricted by professional knowledge and legal regulations, so the lack of annotated corpora becomes one of the greatest technical challenges. At present, ER attracts a lot of attention from the field to improve the representation learning capability of current methods. Research studies have demonstrated that using embedding techniques can help solve the problem of missing supervised data in NLP tasks, including the factorization methods of Global Vectors (GloVe) [9], the neural methods of word2vec [10] and fastText [11], and more recent dynamic methods that take into account the context, such as Embeddings from Language Models (ELMo) [12] and OpenAI Generative Pre-trained Transformer (GPT) [13]. Those embedding technologies can capture the context of semantics in unsupervised data and generate different vector representations of the same word in different contextual situations.

Among them, Bidirectional Encoder Representations from Transformers (BERT) [14] integrates many top ideas of language models and gives a particularly prominent performance. Transform-block is a feature extractor and learns different types of abstract granularity information. Multi-layer information is iterated layer by layer to generate embedding representation. In the actual training process, most downstream tasks take BERT's last embedding vector as the input of the model. However, studies found that different NLP tasks have different characteristics of requirements. Therefore, combining task features into the language model can reduce the loss of extracted information by the feature extractor and improve the utilization of language models. For example, Peters et al [12] explicitly showed that the lower layer fits into the local semantic relationships, the higher layer is suitable for longer-range relationships, and the final layer specializes in the language model. Peters et al [15] also showed that combining all semantic internal states models, by using a weighted-sum method to represent the vector of a word, can enrich the characteristics of the word in learning deep contextualized embedding representations. Because the Chinese ER task focuses on word granularity information, this is a straightforward way to use the information extracted from the low-layer representation.

In this work, we tackle representation using the BERT language model. Our objective is to extract each layer of semantic information using feature extractors. We constructed a multi-level representation learning model for the optimal integration of information. Our contributions can be summarized as follows:

1. We manually annotated a new Chinese EMR (CEMR) corpus for ER tasks. Moreover, we propose a multi-level representation learning model to mine hidden representation.

2. The proposed model takes advantage of the multi-head attention mechanism to integrate more suitable information from each layer and can perform as a state-of-the-art method on two clinical text datasets.

3. The best F1 score achieved by the experiment was $82.11 \%$ on the CEMR corpus and significant improvement on the CCKS (China Conference on Knowledge Graph and Semantic Computing) 2018 benchmark dataset was attained.

\section{Chinese Electronic Medical Record Dataset: A Newly Constructed Corpus}

Large labeled datasets are not always readily accessible. To facilitate the research on the ER task of the Chinese EMRs and 
future work in related topics, we constructed a new manually annotated CEMR dataset. The normalization of the labeling process refers to a large number of annotation guidelines [16]. All EMRs came from Third-Class A-Level hospitals in Gansu Province, China, which contained 80,000 EMRs across 14 departments. Manual labeling of 4000 medical records provided the data for ER experiments. Table 1 shows the data distribution of the 14 hospital departments. The CEMR corpus contains six types of entities: disease (Dis), symptom (Sym), test, treatment (Tre), medicine (Med), and abnormal inspection result (Abn). The categories are defined as follows:

1. Disease: refers to a specific abnormal pathological condition. This abnormal life condition is caused by disorders of self-regulation, such as diabetes.

2. Symptom: refers to subjective feelings described by patients or objective facts observed externally, such as abdominal distension.
3. Test: includes examination procedures, items, and equipment to collect and confirm more information about the disease or symptom, such as electrocardiogram.

4. Treatment: refers to a treatment program or intervention to treat diseases or relieve symptoms, such as neurotrophic treatment.

5. Medicine: refers to a chemical substance used to prevent and treat diseases or to strengthen the body and improve mental state, such as insulin.

6. Abnormal inspection result: refers to an abnormal change or inspection result observed by doctors or by examination equipment, such as a little sputum sound.

Before labeling the data, private information was removed in the EMRs, such as patients' names, addresses, and hospital IDs. In the process of labeling samples, the annotation tool is developed specifically for the ER task. Moreover, some strategies have been developed to create high-quality annotated data. For example, the annotation samples will be randomly checked at any time.

Table 1. Electronic medical record (EMR) data distribution by department.

\begin{tabular}{ll}
\hline Department & EMR count, $\mathrm{n}(\%)$ \\
\hline Neurosurgery & $77(1.93)$ \\
Neurology & $77(1.93)$ \\
Cardiology & $77(1.93)$ \\
Gynecology and obstetrics & $77(1.93)$ \\
Andrology & $77(1.93)$ \\
Respiratory medicine & $77(1.93)$ \\
Cardiovasology & $77(1.93)$ \\
Hepatobiliary surgery & $77(1.93)$ \\
Ophthalmology & $77(1.93)$ \\
Orthopedics & $77(1.93)$ \\
Gynecology & $101(2.53)$ \\
Pediatrics & $232(5.80)$ \\
Internal medicine & $970(24.25)$ \\
Surgery & $1495(37.38)$ \\
Other & $432(10.80)$ \\
Total & $4000(100)$ \\
\hline
\end{tabular}

\section{Methods}

\section{Overview}

The goal of the ER task is to provide the model with an EMR and its semantic types, so that it can extract and classify all characters in the text. The proposed model consists of three stacked layers: the input layer, the feature extraction layer, and the output layer.

As shown in Figure 2, the model first used the BERT language model to extract the semantic representations. Then, the multi-head attention mechanism was leveraged to automatically extract deeper semantic information from each layer. Finally, the semantic information from the multi-level representation extraction was utilized as the final semantic context embedding for each token and was input into the softmax layer to predict the entity tag. The input sentence was denoted as $\mathrm{C}=\left(\mathrm{c}_{1}, \mathrm{c}_{2}\right.$, $\left.\mathrm{c}_{3}, \ldots, \mathrm{c}_{\mathrm{n}}\right)$, where $\mathrm{c}_{\mathrm{n}}$ represented the $n$-th character in sentence $\mathrm{C}$ of the Chinese EMR. Correspondingly, the output sentence's predicted tag sequence was denoted as $\mathrm{Y}=\left(\mathrm{y}_{1}, \mathrm{y}_{2}, \mathrm{y}_{3} \ldots, \mathrm{y}_{\mathrm{n}}\right)$, where $\mathrm{y}_{\mathrm{n}}$ belonged to one of the sets: B-Dis, I-Dis, B-Sym, I-Sym, B-Test, I-Test, B-Tre, I-Tre, B-Med, I-Med, B-Abn, $\mathrm{I}-\mathrm{Abn}$, or $\mathrm{O}$. In the following text, we introduce the BERT language model and describe the proposed multi-level representation learning model. 
Figure 2. Multi-level representation learning for ER model. B-Sym: beginning of the noun phrase for the symptom entity; B-Test: beginning of the noun phrase for the test entity; $\mathrm{C}$ : input sentence; E: input embedding; I-Sym: middle of the noun phrase for the symptom entity; I-Test: middle of the noun phrase for the test entity; O: not a noun phrase; Trm: transform-block; y: output sentence's predicted tag sequence.

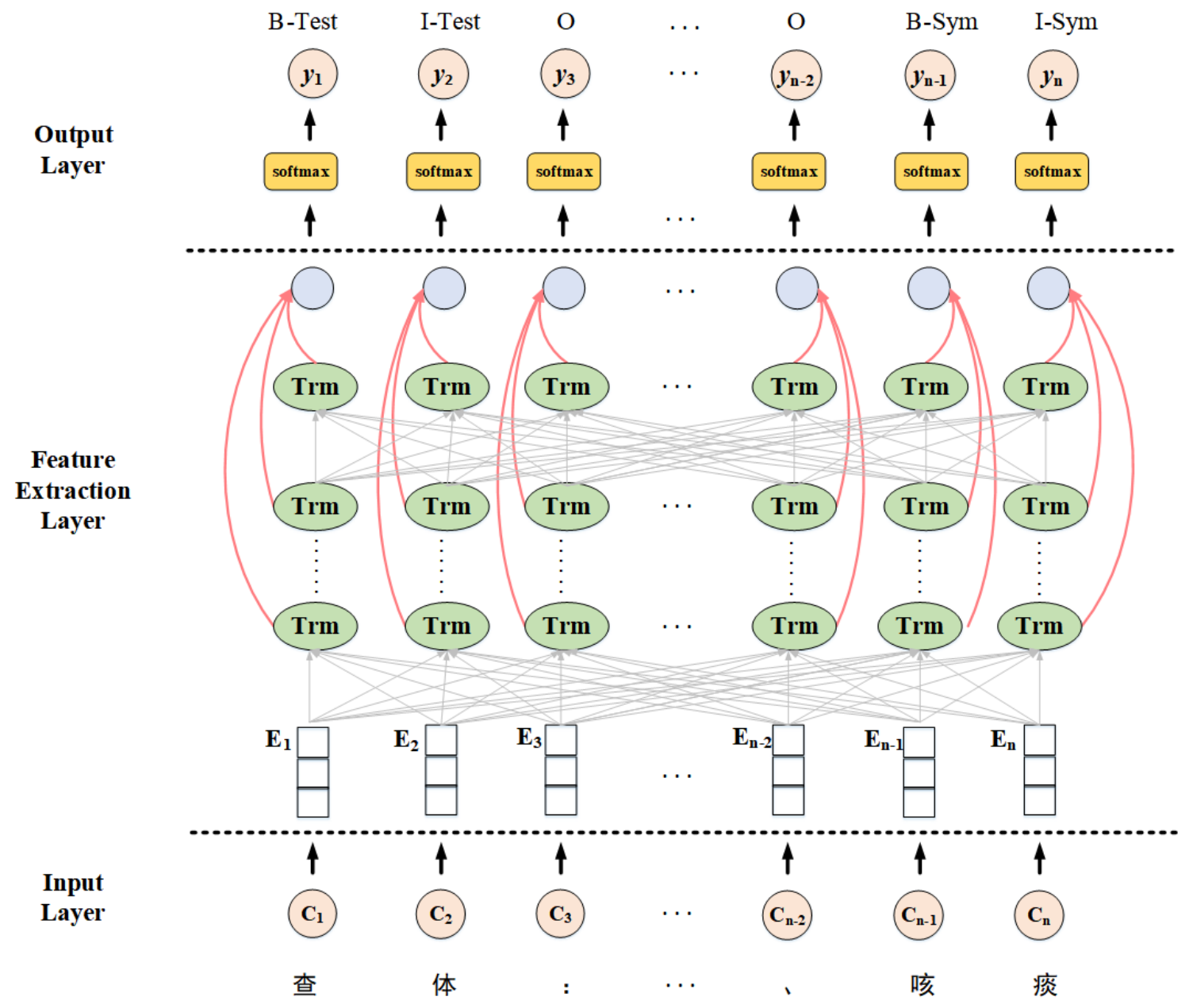

\section{Bidirectional Encoder Representations From Transformers}

BERT was designed to learn deep bidirectional representations by jointly conditioning both the left and right contexts in all layers. It was based on multi-layer bidirectional encoder transformers and could be used for different architectures. When given a character-level sequence $\mathrm{C}=\left(\mathrm{c}_{1}, \mathrm{c}_{2}, \mathrm{c}_{3}, \ldots, \mathrm{c}_{\mathrm{n}}\right)$, BERT was formulated as follows:

$$
\begin{aligned}
& h_{l}=E_{\text {Token }}+E_{\text {Segment }}+E_{\text {Position }}(1) \\
& h_{l}=\operatorname{Trm}\left(h_{l-1}\right)(2) \\
& Y^{B E R T}=\operatorname{Softmax}\left(w_{O} h_{L}+b_{O}\right)(3)
\end{aligned}
$$

where $h_{1}$ represents input embedding for a sequence and is made up of $E_{\text {Token }}, E_{\text {Segment }}$, and $E_{\text {Position }}$, which mean token, segment, and position for a sentence, respectively. The BERT leverage transformer is the feature extractor. Trm is a transform-block that includes self-attention, the fully connected layers, and the output layer. The current $l$ layer hidden state came from the upper $l-l$ layer and $L$ was the last layer. $Y^{B E R T}$ denotes the output layer that predicts the sequence labels. In the above equations, $w_{O}$ denotes the function weight and $b_{O}$ is the function bias. All parameters of the transform-block were trained in advance on a large document-level corpus using a masked language model and were fine-tuned by predicting task-specific labels with the output layer to maximize the log-probability of the correct label.

\section{Multi-Level Representation Learning for Entity Recognition}

The Multi-Level Representation Learning for ER model (Multi-Level ER) could automatically integrate deeper semantic information from all layers of the feature extractor for ER task. The proposed language model took advantage of the multi-head attention mechanism. Multi-head attention is a special type of attention that allowed the model to focus on different positions of subspace representation information and could learn more about the connections between internal elements. Figure 3 shows the calculation process of the multi-head attention mechanism when calculating the weight of the transform-block output knowledge. The query $(\mathrm{Q})$, key $(\mathrm{K})$, and value $(\mathrm{V})$ in the transform-block were calculated. The process of acquiring $\mathrm{Q}$, $\mathrm{K}$, and $\mathrm{V}$ could be written as follows:

$$
\begin{aligned}
& H=\operatorname{Concat}\left(h_{1}, h_{2}, h_{3}, \ldots, h_{L}\right)(4) \\
& Q=w_{Q} h_{L}+b_{L}(5) \\
& K=w_{K} H+b_{K}(6) \\
& V=w_{V} H+b_{V}(7)
\end{aligned}
$$


where $h_{L}$ denotes the hidden state of the final layer of the transform-block. The parameters $w_{Q}, w_{K}$, and $w_{V}$ are weight matrices. The parameters $b_{L}, b_{K}$, and $b_{V}$ are bias matrices. The attention function is calculated as follows:

$$
\operatorname{head}_{i}=\operatorname{Softmax}\left(Q_{L} K^{T} / \sqrt{d}\right) V(8)
$$

where head $_{i}$ means the $i$-th head. $Q_{L}$ is the query key value of the last $L$ layer. $\sqrt{ } d$ is used to control the order of magnitude of calculation results and $d$ donates the dimension of the $K$ vector.

Figure 3. Multi-head attention mechanism. K: key; Q: query; V: value.
In this work, we used multi-head attention, as introduced in the following equation:

$$
E=\text { Concat }\left(\text { head }_{1}, \text { head }_{2}, \text { head }_{3}, \ldots, \text { head }_{l}\right) w_{h}+b_{h}(9)
$$

where $w_{h}$ is used to balance the head weight. For the final layer of the network, we pass the results into a fully connected layer with a softmax function, as follows:

$$
Y^{\text {Multi-Level ER }}=\operatorname{Softmax}\left(w_{O} E+b_{O}\right)(10)
$$

where $w_{O}$ is the output weight matrix and $b_{O}$ is the bias of the output layer.
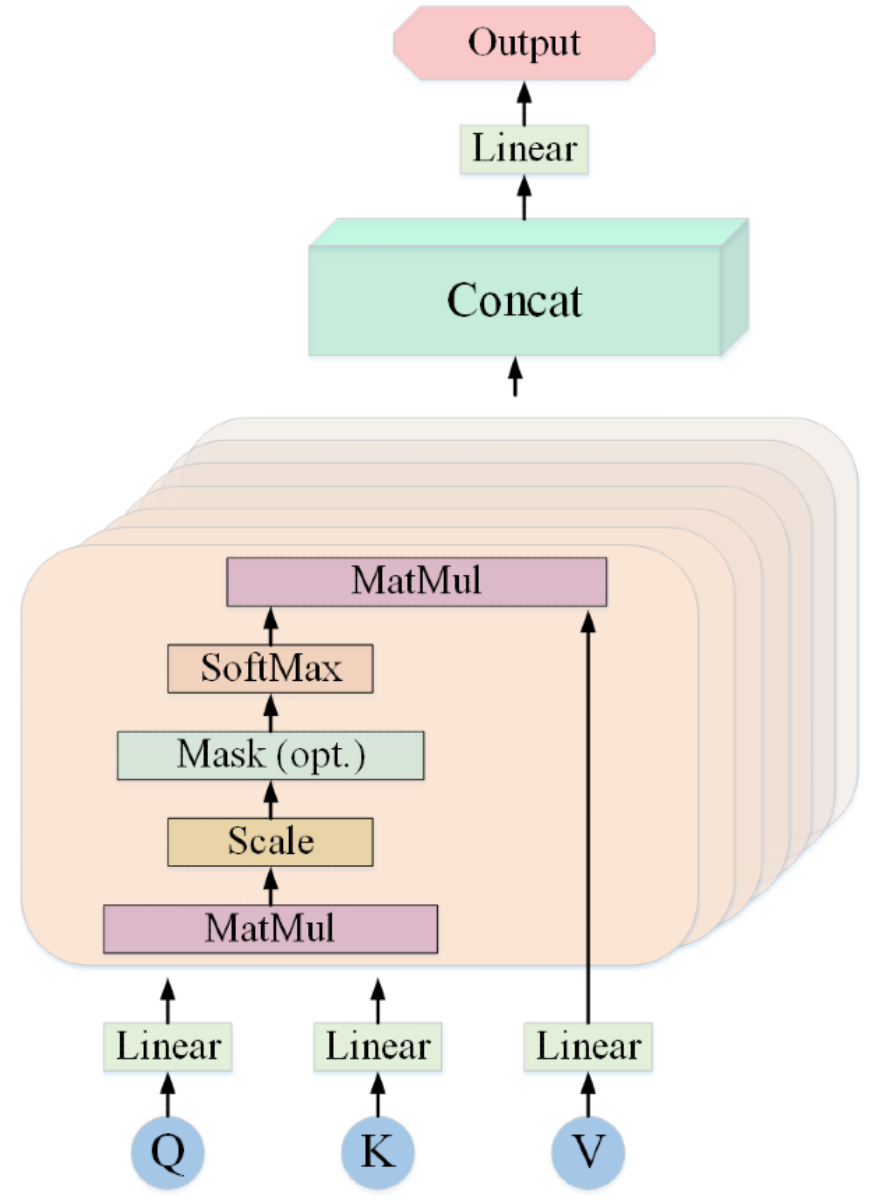

\section{Experiment}

This model was supported by multiple sets of comparative experiments. Each group of experiments was repeated three times, and the result in the middle of the ranking was taken as the final result.

\section{Dataset and Evaluation Criteria}

We evaluated the proposed model on two datasets: the CCKS 2018 dataset and the CEMR dataset. The CCKS 2018 dataset was adopted from the Chinese EMR named ER task at the CCKS, which included 1000 admission records. In the experiment, 600 records were used as training data and the remaining were test data. Comparative experiments were made on the new CEMR corpus and contained 4000 documents. We further split the corpus set by $60 \%, 20 \%$, and $20 \%$ as training, validation, and test sets, respectively. Table 2 shows the distribution of documents in two datasets. 
Table 2. Components of the two datasets.

\begin{tabular}{|c|c|c|c|c|}
\hline \multirow[t]{2}{*}{ Dataset } & \multicolumn{4}{|c|}{ Number of records per set } \\
\hline & Total & Training set & Validation set & Test set \\
\hline CEMR $^{\mathrm{a}}$ dataset & 4000 & 2400 & 800 & 800 \\
\hline $\mathrm{CCKS}^{\mathrm{b}} 2018$ & 1000 & 600 & $\mathrm{~N} / \mathrm{A}^{\mathrm{c}}$ & 400 \\
\hline
\end{tabular}

${ }^{\mathrm{a} C E M R}$ : Chinese electronic medical record.

${ }^{\mathrm{b}} \mathrm{CCKS}$ : China Conference on Knowledge Graph and Semantic Computing.

${ }^{\mathrm{c}}$ Not applicable; because the comparison method does not divide the validation set on the CCKS dataset, we have kept this the same as the original experiment to make the comparison fair.

To evaluate the performance of all prediction methods fairly, the results were validated by precision $(\mathrm{P})$, recall $(\mathrm{R})$, and $\mathrm{F} 1$ scores $(\mathrm{F} 1)$ as measurements to evaluate the recognition effectiveness of the model; these were defined as follows:

$$
\begin{aligned}
& \mathrm{P}=\mathrm{TP} /(\mathrm{TP}+\mathrm{TF})(11) \\
& \mathrm{R}=\mathrm{TP} /(\mathrm{TP}+\mathrm{FN})(12) \\
& \mathrm{F} 1=(2 \times \mathrm{T} \times \mathrm{P}) /(\mathrm{P}+\mathrm{R})(13)
\end{aligned}
$$

An entity is annotated as correct when its category and boundary are fully labeled correctly. TP is the count of entity labels presenting the same labels as gold standard labels, FP is the count of recognized entities marked incorrectly in the results, and $\mathrm{FN}$ is the count of the gold standard entities that are not present in the results of the indicator.

\section{Parameter Setup}

Hyperparameter configuration was adjusted according to the performance on the described validation sets. We used a publicly available pretraining language representation model, namely

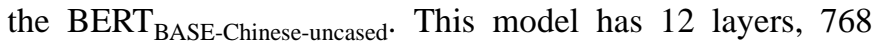
hidden layers, and 12 heads. The multi-head attention mechanism was utilized to automatically integrate all layers of information. By comparing experimental results with different head numbers, we had set the head number to 12 . We fine-tuned the model over 10 epochs with a batch size of 32 . The maximum training sentence length was 64 . The model was trained with the AdamW optimizer with a learning rate of le-5 and we applied a dropout rate of 0.3 .

\section{Results}

\section{Overview}

We summarized the overall performance by computing the F1 score; the results are illustrated in Table 3. On the CEMR dataset, we compared the multi-level ER learning model with previous classic methods, including conditional random field (CRF), convolutional neural network $(\mathrm{CNN})+$ bidirectional long short-term memory (BiLSTM)+CRF, lattice long short-term memory (LSTM), and BERT. We found that the proposed model is better than state-of-the-art baseline methods, with F1 scores of $0.94 \%$ to $4.9 \%$. Our multi-level ER learning model had improved by $1.48 \%$ in its $P$ value, $0.47 \%$ in its $\mathrm{R}$ value, and $0.94 \%$ in its F1 score compared to the BERT model. The result also demonstrated that pretraining the multi-level ER learning language model was highly effective for task-specific Chinese EMR ER.

Table 3. Comparison of method performance on the Chinese electronic medical record (CEMR) dataset.

\begin{tabular}{llll}
\hline Method & $P$ value (\%) & R value (\%) & F1 score (\%) \\
\hline Conditional random field (CRF) & 88.57 & 68.43 & 77.21 \\
CNN $^{\mathrm{a}}+$ BiLSTM \\
berF & 81.51 & 76.92 & 79.15 \\
Lattice long short-term memory (LSTM) & 88.60 & 74.48 & 80.93 \\
Bidirectional Encoder Representations from Transformers (BERT) & 83.73 & 78.76 & 81.17 \\
Multi-level representation learning for entity recognition (multi-level ER) & 85.21 & 79.23 & 82.11 \\
\hline
\end{tabular}

${ }^{\mathrm{a}} \mathrm{CNN}$ : convolutional neural network.

${ }^{\mathrm{b}}$ BiLSTM: bidirectional long short-term memory.

We also applied our model to the widely used benchmark CCKS 2018 dataset and used the same data split to compare it. Huang et al [17] proposed a BiLSTM-CRF model for sequence tagging and Cai et al [18] was based on the self-matching attention mechanism (SM) and proposed an SM-LSTM-CRF model design for the named ER task. The results are shown in Table 4. Under the condition of not needing any external resources, the proposed multi-level ER learning model already outperformed the previous SM-LSTM-CRF model by $3.1 \%$ on the F1 score. 
Table 4. Comparison of method performance on the China Conference on Knowledge Graph and Semantic Computing 2018 dataset.

\begin{tabular}{llll}
\hline Method & $P$ value (\%) & R value (\%) & F1 score (\%) \\
\hline BiLSTM $^{\mathrm{a}}$-CRF ${ }^{\mathrm{b}}[17]$ & 65.68 & 69.04 & 67.32 \\
SM $^{\mathrm{c}}$-LSTM-CRF [18] & 80.54 & 79.61 & 80.08 \\
$\begin{array}{l}\text { Multi-level representation learning for entity recognition (multi- } \\
\text { level ER) }\end{array}$ & 83.90 & 82.47 & 83.18 \\
\hline
\end{tabular}

${ }^{\mathrm{a} B i L S T M}$ : bidirectional long short-term memory.

${ }^{\mathrm{b}} \mathrm{CRF}$ : conditional random field.

${ }^{\mathrm{c}} \mathrm{SM}$ : self-matching attention mechanism.

\section{The Effect of Assembling Methods}

We compared the effects of different assembling methods on model performance to verify the ability of the multi-head attention mechanism to combine hierarchical information. As listed in Table 5, we first applied concatenation that directed the horizontal concatenated tensors; the F1 score was $81.51 \%$. We then adopted the sum average method to get an F1 score of
$81.11 \%$. We finally adopted the multi-head attention method, given that it had the best overall performance compared to several other methods we evaluated. The results showed that integrated hidden information can acquire more suitable representation; the multi-head attention mechanism can be leveraged to automatically extract deeper semantic information from each layer, which is the most effective assembling method.

Table 5. The effect of assembling methods.

\begin{tabular}{llll}
\hline Assembling method & $P$ value $(\%)$ & R value $(\%)$ & F1 score $(\%)$ \\
\hline Concatenation & 84.22 & 78.97 & 81.51 \\
Sum average & 83.27 & 79.06 & 81.11 \\
Multi-head attention mechanism & 85.21 & 79.23 & 82.11 \\
\hline
\end{tabular}

\section{The Effect of Extraction Layer Numbers}

To examine the impact of extraction layer numbers on model performance, we performed comparative experiments using various extraction layer numbers; the results are shown in Table 6. It was observed that the performance of all layers was superior to that of the other numbers of layers, which introduced multi-level ER into the language model and enhanced model performance. By and large, the tendency was that performance improved as the number of extracting layers increased. However, we also discovered that extracting the last four layers gave higher F1 scores than extracting the last six or two layers. The analysis showed that the results were closely related to the specific dataset. Of course, as the number of layers increased, parameters required by the neural network also increased significantly. Therefore, when there was a high demand for speed on the model, we could select a structure that included the last four layers to optimize time efficiency.

Table 6. The effect of extracted layer numbers.

\begin{tabular}{llll}
\hline Extraction layer number & $P$ value $(\%)$ & R value $(\%)$ & F1 score $(\%)$ \\
\hline Total layers & 85.21 & 79.23 & 82.11 \\
The last six layers & 85.15 & 78.65 & 81.77 \\
The last four layers & 85.50 & 78.68 & 81.95 \\
The last two layers & 84.51 & 78.68 & 81.49 \\
\hline
\end{tabular}

\section{The Effect of Dataset Size}

Figure 4 shows the impact of the dataset size on model performance. Horizontal coordinates represent the size of the training dataset and vertical coordinates indicate the F1 scores. During the experiment, we used different sized corpora to train the CNN-LSTM-CRF, BERT, and multi-level ER models. The figure shows that as the training dataset increased, the performance of the models also improved. In reality, we had a limited number of datasets, and models were unlikely to reach saturation. Therefore, the impact of dataset size on performance was particularly critical. We found that the CNN-LSTM-CRF model performance was sharply affected by the size of the dataset when the training set increased from $70 \%$ to $100 \%$. Inversely, the BERT model and the multi-level ER model were less influenced by the training dataset size, and our proposed multi-level RE model outperformed the BERT model. 
Figure 4. The effect of dataset size. BERT: Bidirectional Encoder Representations from Transformers; CNN: convolutional neural network; CRF: conditional random field; LSTM: long short-term memory; Multi-Level ER: multi-level representation learning for entity recognition.

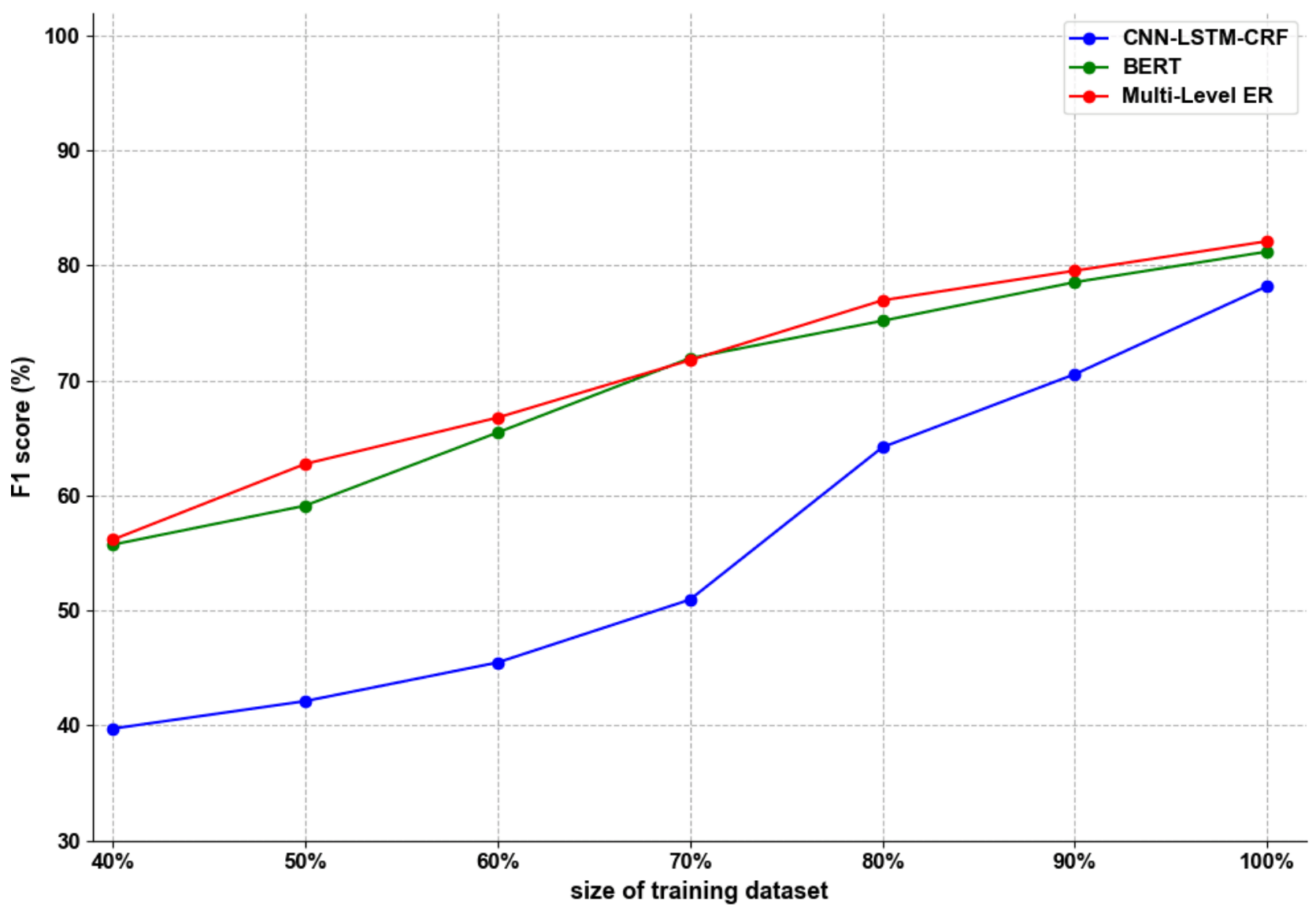

\section{Discussion}

\section{Case Studies}

To show that our model was able to solve the challenge of integrating representation information, three case studies comparing the multi-level ER model with the BERT model are shown in Figure 5. Several obvious trends emerged from the comparative experiments. Most generally, when the word "disease" is included within the medical history, it is mistaken for a disease. For example, case study 1 in Figure 5 shows that "history of mental disease" is recognized as a disease. Case study 2 in Figure 5 shows that when "anal" and "external genitals" appear together before the examination, the system will only identify the adjacent area to be tested. The descriptions with the obvious word "treatment" are identified as a treatment in case study 3 of Figure 5.

Figure 5. Case studies comparing the multi-level representation learning for entity recognition (Multi-Level ER) model with the Bidirectional Encoder Representations from Transformers (BERT) model.

\begin{tabular}{|c|c|c|c|}
\hline Case & BERT & Multi-Level ER & Correct Entity \\
\hline \multirow{2}{*}{ Case 1} & 否认糖尿病、胸血管疾病、精神疾病史。 & 否认糖尿病、胸血管疾病、精神疾病史。 & 否认糖尿病、胸血管疾病、精神疾病史。 \\
\hline & $\begin{array}{l}\text { Deny diabetes, cer ebrovascular disease, and } \\
\text { history of mental disease. }\end{array}$ & $\begin{array}{l}\text { Deny diabetes, cer ebrovascular disease, and } \\
\text { history of mental disease. }\end{array}$ & $\begin{array}{l}\text { Deny diabetes, cer ebrovascular disease, and } \\
\text { history of mental disease. }\end{array}$ \\
\hline \multirow{2}{*}{ Case 2} & 肚门及外生殖器检查结果：无异常。 & 肚门及外生殖器检查结果：无异常。 & 肚门及外生殖器检查结果：无异常。 \\
\hline & $\begin{array}{l}\text { Anal and external genital examination results: no } \\
\text { abnormal. }\end{array}$ & $\begin{array}{l}\text { Anal and external genital examination results: no } \\
\text { abnormal. }\end{array}$ & $\begin{array}{l}\text { Anal and external genital examination results: no } \\
\text { abnormal. }\end{array}$ \\
\hline \multirow{2}{*}{ Case 3} & 今悉者及家属为求进一步治疗。 & 今悉者及家属为求进一步治序。 & 今靑者及家属为求进一步治疗。 \\
\hline & $\begin{array}{l}\text { The patient and his family sought further } \\
\text { treatment. }\end{array}$ & $\begin{array}{l}\text { The patient and his family sought further } \\
\text { treatment. }\end{array}$ & $\begin{array}{l}\text { The patient and his family sought further } \\
\text { treatment. }\end{array}$ \\
\hline
\end{tabular}

We found that the BERT model's embedding technology improves the performance of the ER model in Chinese EMRs; however, using information from only the last layer of the feature extractor in the language model did not achieve the best experimental results. Our proposed multi-level ER model combines the information from each layer of the feature extractor and selects the most suitable, long-term, syntactic, relationship information for the ER task, which greatly improves the performance of the model. 


\section{Related Work}

ER tasks attract a large amount of scholastic attention. The development of deep learning methods has resulted in a breakthrough regarding these tasks. CNN and recurrent neural network (RNN) models have emerged one after another; the attention mechanism and transfer learning were applied to the model. Wu et al [19] utilized a CNN model to generate features represented by several global hidden nodes. Both local features and global features were then fed into a standard affine network to recognize named entities in clinical text. Ju et al [20] used an LSTM neural model to identify nested entities by dynamically stacking flat, named ER layers. Rei et al [21] applied the attention mechanism to dynamically decide how much information to use from a character-level or word-level component in an end-to-end model. Lee et al [22] applied transfer learning in named ER by training a model on source task and using the trained model on the target task for fine-tuning. Peng et al [23] proposed a method where the prediction model was based on BiLSTM, which was taken as the source task of transfer learning. For the ER task in clinical notes, Bharadwaj et al's [24] work centered on effectively adapting these neural architectures toward low-resource settings using parameter transfer methods.

Language models can capture the syntactic and semantic information of words from a large number of unlabeled texts, which alleviates the problem of an insufficiently annotated corpus in special domains. Peters et al [12] used a language model to obtain a deep contextualized word pretraining representation called ELMo and improved the accuracy of six NLP tasks. Radford et al [13] proposed the GPT for language understanding tasks. For text classification and sequence labeling tasks, the transfer ability is better. Devlin et al [14] proposed the pretraining of deep bidirectional transformers for language understanding (ie, BERT); it captured true directional context information, sweeping 11 NLP tasks through pretraining and fine-tuning.

Our motivation is to seize the optimal information from each layer of a feature extractor to suit a given task. Takase et al [25] employed intermediate layer representation, including input embedding, to calculate the probability distributions to solve a ranking problem in language generation tasks. Kaneko et al [26] demonstrated that learning suitable representation came from different layers in grammatical error detection tasks. Therefore, we tracked their work and found the issue in the ER task in Chinese EMRs.

\section{Conclusions}

We propose a novel, multi-level, representation learning model for ER of Chinese EMRs-the multi-level ER model. We compared our model with state-of-the-art models and observed comparable performance without any external syntactic tools. The results showed that the use of the multi-head attention mechanism can effectively integrate deep semantic information from each layer of the feature extractor. In the future, we plan to apply multi-level ER to other language representation models in order to obtain even greater improvement.

\section{Acknowledgments}

This work is supported by the National Natural Science Foundation of China (No. 61762081, No. 61662067, and No. 61662068) and the Key Research and Development Project of Gansu Province (No. 17YF1GA016). The datasets used and analyzed during this study are available from the first author upon reasonable request. The CCKS 2018 dataset that supports the findings of this study were adopted from the Chinese EMR named ER task from the CCKS 2018, but restrictions apply to the availability of these data, which were used under license for this study and are not publicly available.

\section{Conflicts of Interest}

None declared.

\section{References}

1. Zhao H, Yang Y, Zhang Q, Si L. Improve neural entity recognition via multi-task data selection and constrained decoding. In: Proceedings of the 2018 Annual Conference of the North American Chapter of the Association for Computational Linguistics: Human Language Technologies (NAACL-HLT 2018). 2018 Presented at: 2018 Annual Conference of the North American Chapter of the Association for Computational Linguistics: Human Language Technologies (NAACL-HLT 2018); June 1-6, 2018; New Orleans, LA p. 346-351 URL: https://www.aclweb.org/anthology/N18-2056.pdf [doi: $10.18653 / \mathrm{v} 1 / \mathrm{N} 18-2056]$

2. Jiang M, Sanger T, Liu X. Combining contextualized embeddings and prior knowledge for clinical named entity recognition: Evaluation study. JMIR Med Inform 2019 Nov 13;7(4):e14850 [FREE Full text] [doi: 10.2196/14850] [Medline: 31719024]

3. Lample G, Ballesteros M, Subramanian S. Neural architectures for named entity recognition. In: Proceedings of the 2016 North American Chapter of the Association for Computational Linguistics: Human Language Technologies (NAACL-HLT 2016). 2016 Presented at: 2016 North American Chapter of the Association for Computational Linguistics: Human Language Technologies (NAACL-HLT 2016); June 12-17, 2016; San Diego, CA p. 260-270 URL: https://www.aclweb.org/anthology/ N16-1030.pdf [doi: 10.18653/v1/N16-1030]

4. Ma X, Hovy E. End-to-end sequence labeling via bi-directional LSTM-CNNs-CRF. In: Proceedings of the 54th Annual Meeting of the Association for Computational Linguistics. 2016 Presented at: 54th Annual Meeting of the Association for Computational Linguistics; August 7-12, 2016; Berlin, Germany p. 1064-1074 URL: https://www.aclweb.org/anthology/ P16-1101.pdf [doi: 10.18653/v1/P16-1101] 
5. Yang Z, Salakhutdinov R, William WC. Transfer learning for sequence tagging with hierarchical recurrent networks. In: Proceedings of the 5th International Conference on Learning Representations (ICLR 2017). 2017 Presented at: 5th International Conference on Learning Representations (ICLR 2017); April 24-26, 2017; Toulon, France p. 1-10 URL: https://arxiv.org/pdf/1703.06345.pdf

6. Lee K, He L, Lewis M, Zettlemoyer L. End-to-end neural coreference resolution. In: Proceedings of the 2017 Conference on Empirical Methods in Natural Language Processing. 2017 Presented at: 2017 Conference on Empirical Methods in Natural Language Processing; September 7-11, 2017; Copenhagen, Denmark p. 188-197 URL: https://www.aclweb.org/ anthology/D17-1018.pdf [doi: 10.18653/v1/D17-1018]

7. Chen X, Shi Z, Qiu X, Huang X. Adversarial multi-criteria learning for Chinese word segmentation. In: Proceedings of the 55th Annual Meeting of the Association for Computational Linguistics. 2017 Presented at: 55th Annual Meeting of the Association for Computational Linguistics; July 30-August 4, 2017; Vancouver, Canada p. 1193-1203 URL: https://www. aclweb.org/anthology/P17-1110.pdf [doi: 10.18653/v1/P17-1110]

8. El Boukkouri H, Ferret O, Lavergne T, Zweigenbaum P. Embedding strategies for specialized domains: Application to clinical entity recognition. In: Proceedings of the 57th Annual Meeting of the Association for Computational Linguistics: Student Research Workshop. 2019 Presented at: 57th Annual Meeting of the Association for Computational Linguistics: Student Research Workshop; July 28-August 2, 2019; Florence, Italy p. 295-301 URL: https://www.aclweb.org/anthology/ P19-2041.pdf [doi: 10.18653/v1/P19-2041]

9. Pennington J, Socher R, Manning C. GloVe: Global Vectors for word representation. In: Proceedings of the 2014 Conference on Empirical Methods in Natural Language Processing (EMNLP). 2014 Presented at: 2014 Conference on Empirical Methods in Natural Language Processing (EMNLP); October 25-29, 2014; Doha, Qatar p. 1532-1543 URL: https://www. aclweb.org/anthology/D14-1162.pdf

10. Mikolov T, Chen K, Corrado G, Dean J. Efficient estimation of word representations in vector space. In: Proceedings of the International Conference on Learning Representations 2013. 2013 Presented at: International Conference on Learning Representations 2013; May 2-4, 2013; Scottsdale, Arizona p. 1-12 URL: https://arxiv.org/pdf/1301.3781.pdf

11. Bojanowski P, Grave E, Joulin A, Mikolov T. Enriching word vectors with subword information. Trans Assoc Comput Linguist 2017 Dec;5:135-146 [FREE Full text] [doi: 10.1162/tacl a 00051]

12. Peters M, Neumann M, Zettlemoyer L, Yih W. Dissecting contextual word embeddings: Architecture and representation. In: Proceedings of the 2018 Conference on Empirical Methods in Natural Language Processing. 2018 Presented at: 2018 Conference on Empirical Methods in Natural Language Processing; October 31-November 4, 2018; Brussels, Belgium p. 1499-1509 URL: https://www.aclweb.org/anthology/D18-1179.pdf [doi: 10.18653/v1/D18-1179]

13. Radford A, Narasimhan K, Salimans T, Sutskever I. OpenAI preprint. 2018. Improving language understanding by generative pre-training URL: https://cdn.openai.com/research-covers/language-unsupervised/language understanding paper.pdf [accessed 2020-04-12]

14. Devlin J, Chang MW, Lee K, Toutanova K. arXiv. 2018. Bert: Pre-training of deep bidirectional transformers for language understanding URL: https://arxiv.org/pdf/1810.04805.pdf [accessed 2019-10-18]

15. Peters M, Neumann M, Iyyer M, Gardner M, Clark C, Lee L, et al. Deep contextualized word representations. In: Proceedings of the 2018 Annual Conference of the North American Chapter of the Association for Computational Linguistics: Human Language Technologies (NAACL-HLT 2018). 2018 Presented at: 2018 Annual Conference of the North American Chapter of the Association for Computational Linguistics: Human Language Technologies (NAACL-HLT 2018); June 1-6, 20188; New Orleans, LA p. 2227-2237 URL: https://www.aclweb.org/anthology/N18-1202.pdf [doi: 10.18653/v1/n18-1202]

16. Stubbs A, Uzuner Ö. Annotating risk factors for heart disease in clinical narratives for diabetic patients. J Biomed Inform 2015 Dec;58 Suppl:S78-S91 [FREE Full text] [doi: 10.1016/j.jbi.2015.05.009] [Medline: 26004790]

17. Huang Z, Xu W, Yu K. arXiv. 2015. Bidirectional LSTM-CRF models for sequence tagging URL: https://arxiv.org/pdf/ 1508.01991v1.pdf [accessed 2019-10-18]

18. Cai X, Dong S, Hu J. A deep learning model incorporating part of speech and self-matching attention for named entity recognition of Chinese electronic medical records. BMC Med Inform Decis Mak 2019 Apr 09;19(Suppl 2):65 [FREE Full text] [doi: 10.1186/s12911-019-0762-7] [Medline: 30961622]

19. $\mathrm{Wu}$ Y, Jiang M, Lei J, Xu H. Named entity recognition in Chinese clinical text using deep neural network. Stud Health Technol Inform 2015;216:624-628 [FREE Full text] [Medline: 26262126]

20. Ju M, Miwa M, Ananiadou S. A neural layered model for nested named entity recognition. In: Proceedings of the 2018 Annual Conference of the North American Chapter of the Association for Computational Linguistics: Human Language Technologies (NAACL-HLT 2018). 2018 Presented at: 2018 Annual Conference of the North American Chapter of the Association for Computational Linguistics: Human Language Technologies (NAACL-HLT 2018); June 1-6, 2018; New Orleans, LA p. 1446-1459 URL: https://www.aclweb.org/anthology/N18-1131.pdf [doi: 10.18653/v1/N18-1131]

21. Rei M, Crichton GKO, Pyysalo S. Attending to characters in neural sequence labeling models. In: Proceedings of the 26th International Conference on Computational Linguistics (COLING 2016): Technical Papers. 2016 Presented at: 26th International Conference on Computational Linguistics (COLING 2016): Technical Papers; December 11-17, 2016; Osaka, Japan p. 309-318 URL: https://www.aclweb.org/anthology/C16-1030.pdf 
22. Lee JY, Dernoncourt F, Szolovits P. arXiv. 2017. Transfer learning for named-entity recognition with neural networks URL: https://arxiv.org/pdf/1705.06273.pdf [accessed 2019-10-18]

23. Peng D, Wang Y, Liu C, Chen Z. TL-NER: A transfer learning model for Chinese named entity recognition. Inf Syst Front 2019 Jun 4:1. [doi: 10.1007/s10796-019-09932-y]

24. Bharadwaj A, Mortensen D, Dyer C, Carbonell J. Phonologically aware neural model for named entity recognition in low resource transfer settings. In: Proceedings of the 2016 Conference on Empirical Methods in Natural Language Processing. 2016 Presented at: 2016 Conference on Empirical Methods in Natural Language Processing; November 1-5, 2016; Austin, TX p. 1462-1472 URL: https://www.aclweb.org/anthology/D16-1153.pdf [doi: 10.18653/v1/D16-1153]

25. Takase S, Suzuki J, Nagata M. Direct output connection for a high-rank language model. In: Proceedings of the 2018 Conference on Empirical Methods in Natural Language Processing. 2018 Presented at: 2018 Conference on Empirical Methods in Natural Language Processing; October 31-November 4, 2018; Brussels, Belgium p. 4599-4609 URL: https:/ /www.aclweb.org/anthology/D18-1489.pdf [doi: 10.18653/v1/D18-1489]

26. Kaneko M, Komachi M. arXiv. 2019. Multi-head multi-layer attention to deep language representations for grammatical error detection URL: https://arxiv.org/pdf/1904.07334.pdf [accessed 2019-10-18]

\author{
Abbreviations \\ Abn: abnormal inspection result \\ BERT: Bidirectional Encoder Representations from Transformers \\ BiLSTM: bidirectional long short-term memory \\ BIO: beginning of the noun phrase, middle of the noun phrase, and not a noun phrase \\ CCKS: China Conference on Knowledge Graph and Semantic Computing \\ CEMR: Chinese electronic medical record \\ CNN: convolutional neural network \\ CRF: conditional random field \\ Dis: disease \\ ELMo: Embeddings from Language Models \\ EMR: electronic medical record \\ ER: entity recognition \\ F1: F1 score \\ GloVe: Global Vectors \\ GPT: Generative Pretraining Transformer \\ K: key \\ LSTM: long short-term memory \\ Med: medicine \\ multi-level ER: multi-level representation learning for entity recognition \\ NLP: natural language processing \\ P: precision \\ Q: query \\ R: recall \\ RNN: recurrent neural network \\ SM: self-matching attention mechanism \\ Sym: symptom \\ Tre: treatment \\ V: value
}

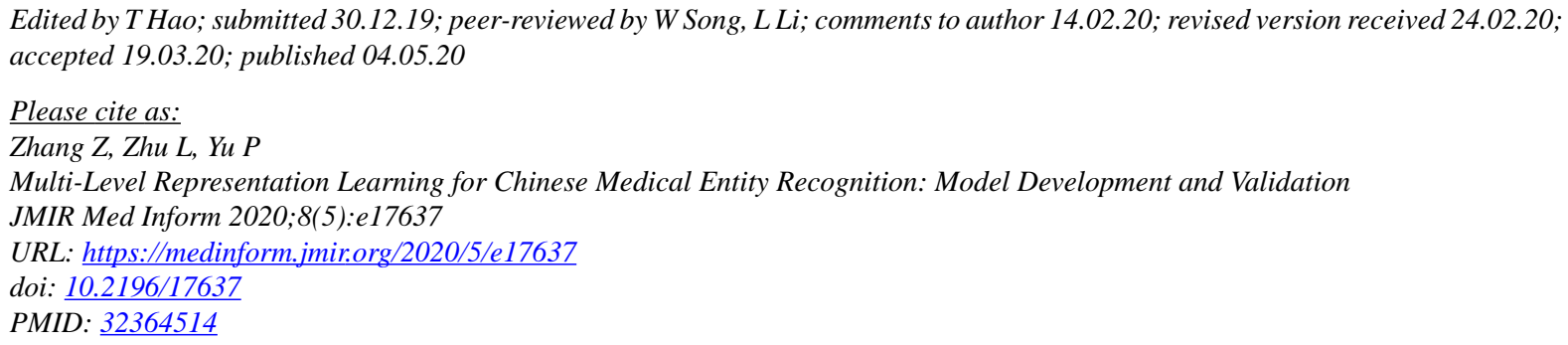


(CZhichang Zhang, Lin Zhu, Peilin Yu. Originally published in JMIR Medical Informatics (http://medinform.jmir.org), 04.05.2020. This is an open-access article distributed under the terms of the Creative Commons Attribution License (https://creativecommons.org/licenses/by/4.0/), which permits unrestricted use, distribution, and reproduction in any medium, provided the original work, first published in JMIR Medical Informatics, is properly cited. The complete bibliographic information, a link to the original publication on http://medinform.jmir.org/, as well as this copyright and license information must be included. 